\title{
Assessing test-retest reliability of patient-reported outcome measures using intraclass correlation coefficients: recommendations for selecting and documenting the analytical formula
}

\author{
Shanshan Qin ${ }^{1} \cdot$ Lauren Nelson $^{1} \cdot$ Lori McLeod $^{1} \cdot$ Sonya Eremenco ${ }^{2} \cdot$ Stephen Joel Coons $^{2}$
}

Accepted: 29 November 2018 / Published online: 13 December 2018

(c) The Author(s) 2018

\begin{abstract}
Purpose The US Food and Drug Administration (FDA) 2009 guidance for industry on patient-reported outcome (PRO) measures describes how the Agency evaluates the psychometric properties of measures intended to support medical product labeling claims. An important psychometric property is test-retest reliability. The guidance lists intraclass correlation coefficients (ICCs) and the assessment time period as key considerations for test-retest reliability evaluations. However, the guidance does not provide recommendations regarding ICC computation, nor is there consensus within the measurement literature regarding the most appropriate ICC formula for test-retest reliability assessment. This absence of consensus emerged as an issue within Critical Path Institute's PRO Consortium. The purpose of this project was to generate thoughtful and informed recommendations regarding the most appropriate ICC formula for assessing a PRO measure's test-retest reliability.

Methods Literature was reviewed and a preferred ICC formula was proposed. Feedback on the chosen formula was solicited from psychometricians, biostatisticians, regulators, and other scientists who have collaborated on PRO Consortium initiatives. Results and conclusions Feedback was carefully considered and, after further deliberation, the proposed ICC formula was confirmed. In conclusion, to assess test-retest reliability for PRO measures, the two-way mixed-effect analysis of variance model with interaction for the absolute agreement between single scores is recommended.
\end{abstract}

Keywords ICC $\cdot$ Intraclass correlation coefficient $\cdot$ PRO $\cdot$ Patient-reported outcome measures $\cdot$ Test-retest reliability

The US Food and Drug Administration (FDA) 2009 guidance for industry on patient-reported outcome (PRO) measures describes how the agency will review and evaluate the development and psychometric properties of measures intended to support medical product labeling claims [1]. Within the psychometric measurement section of the guidance, a key property for review is test-retest reliability, defined as the "stability of scores over time when no change is expected in the concept of interest." The guidance also lists intraclass correlation coefficients (ICCs) and the time period of assessment as key considerations in FDA review of the test-retest reliability evaluations. While the guidance

Shanshan Qin

sqin@rti.org

1 RTI Health Solutions, 3040 Cornwallis Road, Research Triangle Park, NC 27709, USA

2 Critical Path Institute, 1730 E River Road, Tucson, AZ 85718, USA describes a number of factors to consider when identifying the time period most appropriate for assessments (e.g., variability of the disease state, reference period of the measure), it does not provide specific recommendations regarding the computation of ICCs.

Within the measurement literature, a variety of computational methods have been used to calculate ICCs, a finding that is further complicated by the use of different notation systems for documenting the selected ICC formula [2-7]. The lack of a consistent approach has resulted in confusion regarding which ICC formula is appropriate for assessing test-retest reliability and the inability to compare ICC results across PRO measures when different formulas are used. This absence of consensus regarding the most appropriate ICC formula specific for the assessment of test-retest reliability in PRO measurement and the lack of a uniform naming convention for the ICC formulas emerged as an issue within Critical Path Institute's (C-Path's) PRO Consortium [8]. C-Path is an independent, nonprofit organization established in 2005 with public and private support to bring together 
scientists and others from regulatory agencies, industry, patient groups, and academia to collaborate on improving the medical product development process. C-Path, in cooperation with FDA and the pharmaceutical industry, formed the PRO Consortium in 2008 to facilitate precompetitive collaboration aimed at advancing the assessment of patientreported and other patient-focused clinical outcomes in drug treatment trials. There was a realization that different, often unidentified, ICC formulas were used by the PRO Consortium's working groups to evaluate the test-retest reliability of its developmental PRO measures without a clear rationale. This made comparison of test-retest reliability among the measures problematic and, ultimately, complicated regulatory submissions due to the absence of a coherent and consistent approach to ICC formula selection.

To address these issues, the authors reviewed the literature and developed recommendations for the most appropriate ICC formula to fulfill their test-retest reliability objective along with the rationale for the recommendations. The draft of this document was provided to a group of twelve experts including psychometricians, biostatisticians, regulators, and other scientists representing the PRO Consortium, the pharmaceutical industry, clinical research organizations, and consulting firms for review and comment. Feedback was received in written form, followed by discussion with some of the experts for further input and clarification. The authors considered the group's input in generating the final recommendations presented in this manuscript for selecting the most appropriate ICC formula within the context of assessing the test-retest reliability of PRO measures to support regulatory review.

In the measurement literature, Shrout and Fleiss [5] and McGraw and Wong [6] appear to be the two most cited references for evaluating test-retest reliability. The seminal work of Shrout and Fleiss [5] presented six computational formulas for ICCs. McGraw and Wong [6] expanded the number from 6 to 10 by incorporating more model assumptions, various study designs, and the corresponding analysis of variance (ANOVA) models into the list of considerations for selecting an ICC formula. Because McGraw and Wong [6] offered a more comprehensive treatment of the selection of an ICC formula and a clearer statement of model assumptions, we recommend using their notational system for clarity. However, a key limitation in the general ICC literature is the use of "raters" in the formulas and in the examples, which does not easily translate to the PRO measurement situation where different "time points" rather than different "raters" is the context for the evaluation.

McGraw and Wong present 10 ICC formulas [6, pp 34-35] from which researchers may select based on factors that include the study design (e.g., multiple ratings per subject or multiple subjects per raters), the number of time points, and the intended generalizability of the findings. To assess test-retest reliability for PRO measures, we recommend the two-way mixed-effect ANOVA model with interaction for the absolute agreement between single scores as the preferred ICC formula based on typical study designs (Table 1).

This recommendation is based on the following considerations:

1. The two-way model is recommended over the one-way model because time is a design factor in a typical testretest assessment and the two time points are not interchangeable (i.e., the chronology is important to detect systematic differences such as learning). An ICC computed using the one-way model would underestimate the reliability due to not partitioning the within-patient variability into the time variability and the error term.

2. A mixed-effect model is recommended over a random effect model because, in the former, test and retest time points are prespecified and identical across all study subjects rather than being randomly selected from the population of all possible pairs of time points. In this case, the time effect is considered as fixed.

3. The time-by-subject interaction is assumed to be included in the error term because the interaction cannot be estimated for situations with only one measurement per subject collected at each time point.

4. Absolute agreement is recommended over consistency because subjects are assumed to be stable for the construct of interest across the two time points. Therefore, the systematic differences in the individual scores on the PRO measure over time are of interest.

There are situations where alternative models are more appropriate, however. For example, when the time points for the test-retest assessment could be considered randomly selected (e.g., any two assessments from a number of assessments in the study) in order to generalize the test-retest reliability of the measure beyond the stated context of use, the use of a two-way random effect model is reasonable. In addition, the proposed ICC formula assumes the use of the same mode of data collection for all time points assessed; an alternative ICC formula may be appropriate for the assessment of measurement equivalence between different modes of data collection of the same PRO measure [9].

Note that the ICC $(\mathrm{A}, 1)$ values remain the same no matter which two-way ANOVA model is constructed. However, we advocate for the articulation of model choice because of the different conceptual considerations being implied. There are many such statistical models where model assumption and interpretation are conceptually different, but some statistics or test results could be the same (e.g., univariate repeated measures ANOVA vs. multivariate ANOVA, and Rasch model vs. 1-parameter logistic item response theory model). 


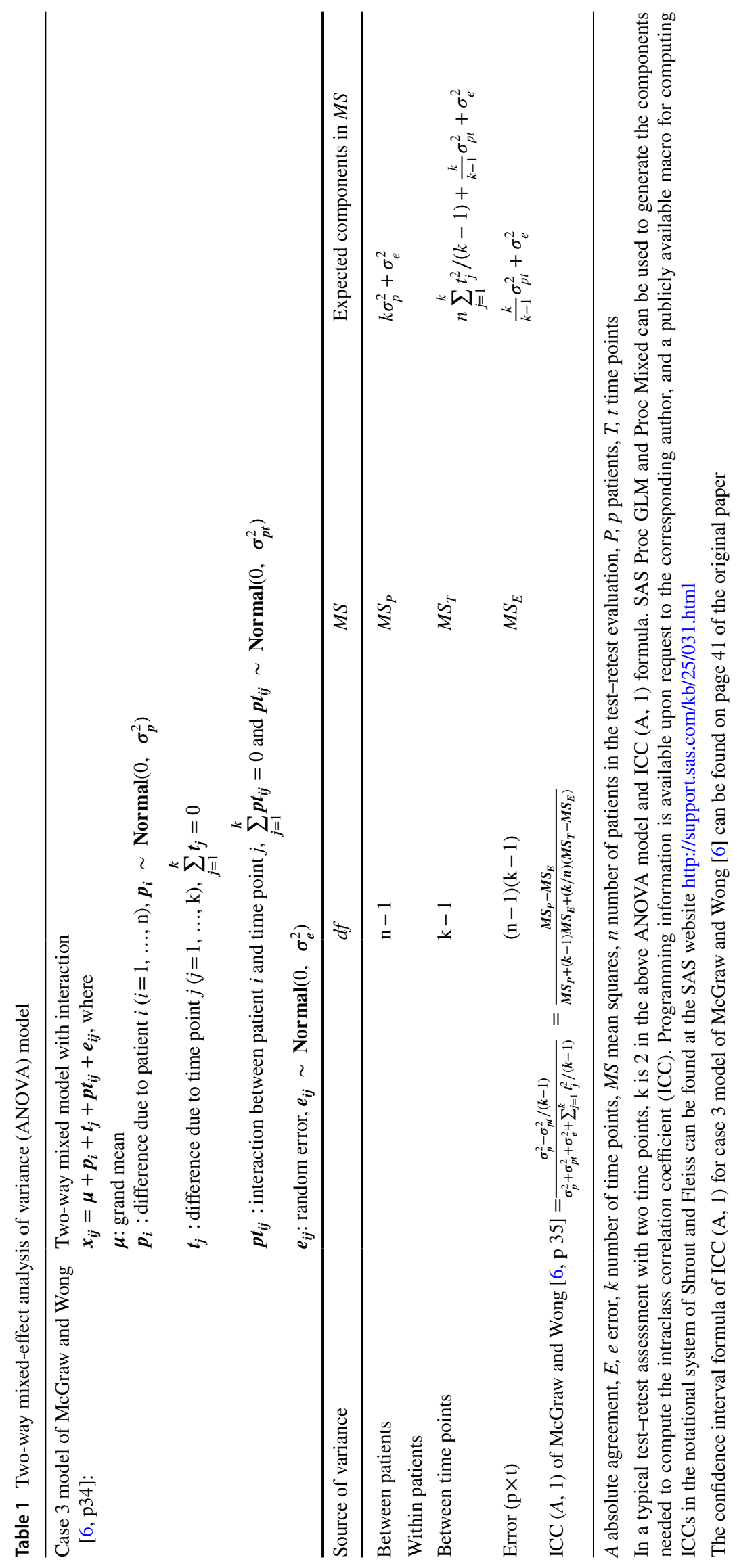




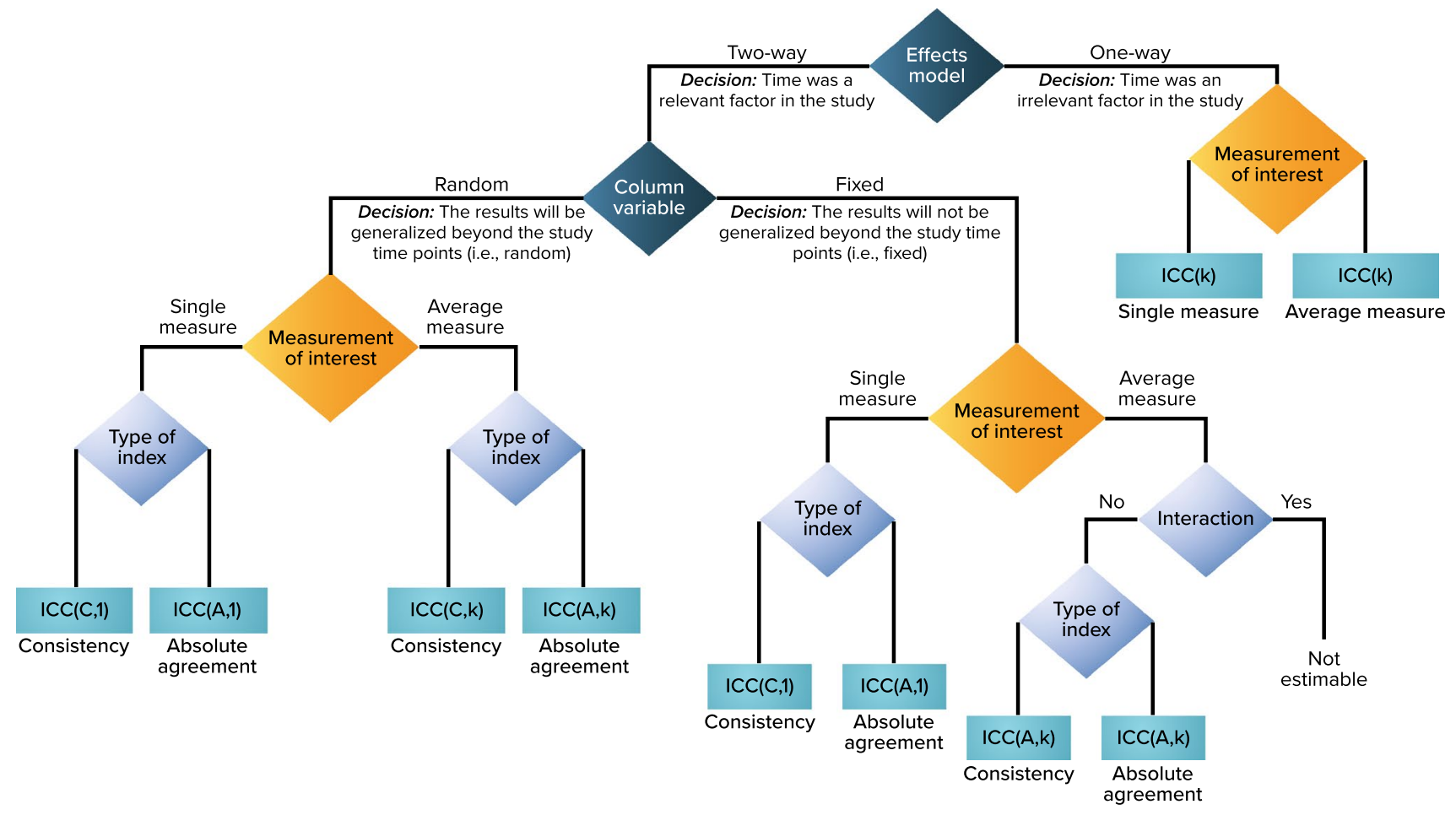

Fig. 1 Test-retest ANOVA Model and ICC Type Decision Flowchart (adapted with permission from [6], American Psychological Association). A absolute agreement, ANOVA analysis of variance, $C$ consist-

We believe that making a clear distinction among models conceptually is important as the chosen model informs the context and the study design. As Schuck [10] noted, "The most important conclusion of the foregoing discussion is not to report 'the' ICC, but to describe which ICC has been used, and for what reason." Whatever the circumstances, we recommend the inclusion of details that describe the exact model used to estimate the ICC and the rationale for the choice. To facilitate the selection of ICC formulas for different study designs (particularly those that are not typical for test-retest reliability evaluation), a decision tree adapted from McGraw and Wong's published decision tree is provided (Fig. 1).

Test-retest ICC values obtained from specific data sets are only point estimates of the true ICC, and they are affected by sample size, data variability, measurement error, and correlation strength as well as by systematic difference between time points $[2,4,6,11]$. In addition to observed ICC values, we recommend always reporting the corresponding confidence intervals to evaluate the precision of the estimate $[6,12,13]$. When unexpected ICC values occur, additional investigations should be conducted to identify potential reasons for the unexpected values. Investigations to consider include the generation of scatter plots and ANOVA tables and/or conduct of additional correlation assessments, t-tests, or subgroup analyses. ency, ICC intraclass correlation coefficient, $k$ average of $\mathrm{k}$ independent measurements

Finally, as ratios of variance components, ICCs of the same model and sample that are calculated using different programming software may vary slightly due to differences in the handling of missing values and the estimation algorithms for variance parameters. Also, due to the fact that between-subject variability is incorporated as part of the ICC ratio, an ICC value is not independent of the study design or specific sample utilized [2]. Low ICC values may be indicative of issues with the study design rather than with the measurement properties of the assessment tool being evaluated. The study population may be restricted to a very narrow subset of scores on the PRO measure's full score range, for example, and thereby restrict between-subject variability. For these reasons and many others, ICC values should be considered as only a single part of the total evidence needed to support the reproducibility of a PRO measure.

Acknowledgements The authors sincerely thank Donald Bushnell, Cheryl Coon, Amylou Dueck, Adam Gater, Stacie Hudgens, R. J. Wirth, and Kathleen Wyrwich for their review and feedback on the first draft of the recommendations made in this paper.

Funding Qin S, Nelson L, and McLeod L were provided support by RTI Health Solutions. Critical Path Institute and Critical Path Institute's Patient-Reported Outcome Consortium are supported, 
in part, by Critical Path Public-Private Partnerships Grant Number U18 FD005320 (effective 2015-2020) from the U.S. Food and Drug Administration.

\section{Compliance with ethical standards}

Conflict of interest Qin S, Nelson L, and McLeod L are researchers employed by RTI-Health Solutions and provide clinical outcome assessment development and psychometric evaluation support for pharmaceutical companies. Eremenco $\mathrm{S}$ and Coons $\mathrm{S}$ are researchers employed by Critical Path Institute and support patient-focused drug development in cooperation with the U.S. Food and Drug Administration's Center for Drug Evaluation and Research and the pharmaceutical industry.

Ethical approval This article does not contain any studies with human participants or animals performed by any of the authors.

Open Access This article is distributed under the terms of the Creative Commons Attribution 4.0 International License (http://creativeco mmons.org/licenses/by/4.0/), which permits unrestricted use, distribution, and reproduction in any medium, provided you give appropriate credit to the original author(s) and the source, provide a link to the Creative Commons license, and indicate if changes were made.

\section{References}

1. Food and Drug Administration. (2009). Guidance for industry on patient-reported outcome measures: Use in medical product development to support labeling claims. Federal Register, 74(235), 65132-65133.

2. Weir, J. P. (2005). Quantifying test-retest reliability using the intraclass correlation coefficient and the SEM. Journal of Strength and Conditioning Research, 19, 231-240.

3. Lee, K., Lee, J., Park, M., et al. (2012). Pitfalls and important issues in testing reliability using intraclass correlation coefficients in orthopaedic research. Clinics in Orthopedic Surgery [serial online ], 4(2), 149-155.
4. Sen, R., Yip, C., \& Severson, K. (2017). The use of intra-class correlation coefficients to assess test-retest reliabilities in psychometric evaluations of patient reported outcome measures. Poster presented at: The International Society for Pharmacoeconomics and Outcomes Research 20th Annual European Congress (November 8). Scotland: 2017; Glasgow.

5. Shrout, P. E., \& Fleiss, J. L. (1979). Intraclass correlations: Uses in assessing rater reliability. Psychological Bulletin, 86, 420-428.

6. McGraw, K. O., \& Wong, S. P. (1996). Forming inferences about some intraclass correlation coefficients. Psychological Methods, 1(1), 30-46.

7. Deyo, R. A., Diehr, P., \& Patrick, D. L. (1991). Reproducibility and responsiveness of health status measures: statistics and strategies for evaluation. Controlled Clinical Trials, 12, 142S-158S.

8. Coons, S. J., Kothari, S., Monz, B. U., \& Burke, L. B. (2011). The Patient-Reported Outcome (PRO) Consortium: Filling measurement gaps for PRO endpoints to support labeling claims. Clinical Pharmacology \& Therapeutics, 90, 743-748.

9. Lundy, J. J., Coons, S. J., \& Aaronson, N. K. (2014). Testing the measurement equivalence of paper and interactive voice response system versions of the EORTC QLQ-C30. Quality of Life Research, 23, 229-237.

10. Schuck, P. (2014). Assessing reproducibility for interval data in health-related quality of life questionnaires: Which coefficient should be used? Quality of Life Research, 13, 571-585.

11. Koo, T. K., \& Li, M. Y. (2016). A guideline of selecting and reporting intraclass correlation coefficients for reliability research. Journal of Chiropractic Medicine, 15(2), 155-163.

12. Zou, K. H., \& McDermott, M. P. (1999). Higher-moment approaches to approximate interval estimation for a certain intraclass correlation coefficient. Statistics in Medicine, 18, 2051-2061.

13. Ionan, A. C., Polley, M. C., et al. (2014). Comparison of confidence interval methods for an intra-class correlation coefficient (ICC). BMC Medical Research Methodology, 14, 121.

Publisher's Note Springer Nature remains neutral with regard to jurisdictional claims in published maps and institutional affiliations. 